\title{
Percepção e expectativas universitárias em tempo da COVID-I 9: um estudo de caso
}

\author{
Perception and university expectations in time of COVID-I 9: \\ a case study
}

\section{Percepción y expectativas universitarias en tiempo de COVID-I 9: \\ un estudio de caso}

\author{
Katia Maria Pacheco dos Santos 1
}

https://orcid.org/000-0003-2942-745

\begin{abstract}
Resumo: A quarentena contra a disseminação da COVID-19 desorganizou o sistema educacional de todos os países, desde a educação infantil ao ensino superior. No mundo inteiro as instituições de ensino superior, a exemplo de outros segmentos da sociedade que suspenderam as atividades letivas presenciais a fim de garantir o isolamento social, assim como foi na Universidade da Integração Internacional da Lusofonia Afro-Brasileira (UNILAB). O objetivo do estudo foi conhecer as percepções e expectativas dos universitários do Curso de Administração Pública da UNILAB a respeito dos efeitos da Pandemia da COVID-19 em suas rotinas sem aulas na Universidade. A coleta de dados se deu por meio de um questionário estruturado elaborado na plataforma virtual Google Forms. Os resultados obtidos poderão auxiliar no planejamento e na tomada de decisão sobre uma futura agenda de atividades acadêmicas.
\end{abstract}

Palavras-chave: Percepção. Educação superior. COVID-19.

Abstract: The quarantine against the spread of COVID-19 disrupted the educational system in all countries, from early childhood education to higher education. Throughout the world, higher education institutions, like other segments of society have suspended classroom activities to ensure social isolation, as happened at the University for the International Integration of Afro-Brazilian Lusophony (UNILAB). The objective of the study was to know the perceptions and expectations of university students in the UNILAB Public Administration Course on the effects of the COVID-I9 pandemic on their routines without classes at the University. Data collection was carried out through a structured questionnaire prepared on the virtual platform of Google Forms. The results obtained can help in planning and decision making in a future academic activities.

Keywords: Perception. College education. COVID-19.

Resumen: La cuarentena contra la propagación de COVID-19 interrumpió el sistema educativo en todos los países, desde la educación de la primera infancia hasta la educación superior. En todo el mundo, las instituciones de educación superior, como otros segmentos de la sociedad, han suspendido las actividades en el aula para garantizar el aislamiento social, tal como sucedió en la Universidad de Integración Internacional de la Lusofonía

\footnotetext{
' Doutora em Ciências. Universidade do Estado do Mato Grosso - UNEMAT. Docente no Campus da UNEMAT em Nova Xavantina-MT. E-mail: katia.maria@unemat.br
}

Olhar de professor, Ponta Grossa, v. 24, p. I-II, e-16I44.052, 2021.

Disponível em <https://revistas2.uepg.br/index.php/olhardeprofessor> 
Afrobrasileña (UNILAB). El objetivo del estudio fue conocer las percepciones y expectativas de los estudiantes universitarios en el Curso de Administración Pública de la UNILAB sobre los efectos de la pandemia COVID-19 en sus rutinas sin clases en la Universidad. La recopilación de datos se realizó a través de un cuestionario estructurado elaborado en la plataforma virtual de Formularios de Google. Los resultados obtenidos pueden ayudar en la planificación y la toma de decisiones en una futura agenda de actividades académicas.

Palabras-clave: Percepción. Educación universitária. COVID-19.

\section{Introdução}

A primeira morte causada pelo novo coronavírus causador da COVID-19 foi anunciada pelas autoridades chinesas em II de janeiro de 2020 (BRASIL, 2020).

Em 30 de janeiro de 2020, a Organização Mundial da Saúde (OMS) declarou o surto de Doença Respiratória Aguda causada por esse novo vírus como uma Emergência de Saúde Pública de Importância Internacional (WHO, 0I/06/2020). Assim, foi dado o alerta a todos os países quanto à necessidade de aplicação de medidas para conter a transmissão desse vírus, bem como a adoção de ações para prevenir a sua disseminação, dentre essas: a vigilância ativa com detecção precoce, o isolamento social, o manejo adequado dos casos, ou seja, investigação/monitoramento dos contatos e a notificação oportuna (RODRIGUEZ-MORALES et al, 2020)

Na América Latina, o primeiro caso da COVID- 19 foi confirmado em 25 de fevereiro de 2020 pelo Ministério da Saúde do Brasil. De acordo com a divulgação do Ministério da Saúde, o primeiro diagnóstico confirmado de pessoa infectada pela COVID-19 foi atestado na cidade de São Paulo, pelo hospital Albert Einstein. Em 12 de março, quando foi reconhecida a transmissão comunitária no estado, São Paulo já registrava 152 casos confirmados da COVID-19, desde então, no Brasil, os números de casos confirmados aumentaram diariamente (SES-SP, 07/04/2020).

Segundo a OMS desde a data do primeiro caso até o dia 07 de abril foram registrados 13.717 casos de pessoas infectadas e 667 mortes no país. Passados dois meses, em 4 de junho, foi contabilizado pela OMS 555.383 casos confirmados de pessoas infectadas pelo coronavírus e 31.199 mortes (WHO, $01 / 06 / 2020)$.

Em todos os estados do Brasil, em consonância com a orientação de especialistas da área de saúde de todas as partes do mundo, bem como da própria OMS, os governos estaduais e municipais adotaram medidas para promover $\mathrm{o}$ isolamento social, uma vez que tal medida é considerada a estratégia mais adequada para conter a propagação do vírus.

No estado do Ceará, em 16 de março de 2020 foi publicado o decreto estadual $n^{\circ} 33.510$ (CEARÁ, 2020), que dita situação de emergência em saúde no Ceará e decreta o isolamento social. Passados quase três meses, em 06 de junho o governo cearense prorroga o isolamento social por meio de outro decreto estadual, o decreto $n^{\circ} 33.617$ (CEARÁ, 2020). 
Segundo Ministério da Saúde, desde o início da pandemia até o dia 13 de junho no estado do Ceará foram contabilizados 76.429 casos de infecção da COVID-19 e 4.829 óbitos (BRASIL, 2020).

É inegável o fato a quarentena contra a disseminação da COVID-19 desorganizou o sistema educacional de todos os países, desde a educação infantil ao ensino superior. No mundo inteiro as instituições de ensino superior, a exemplo de outros segmentos da sociedade, suspenderam as atividades letivas presenciais, a fim de garantir o isolamento social, assim como foi na Universidade da Integração Internacional da Lusofonia Afro-Brasileira - UNILAB.

A UNILAB por meio da portaria reitoria n'II0 (UNILAB, 17/03/2020), instituiu o Comitê Institucional de Enfrentamento a COVID-19, com o objetivo de subsidiar as decisões institucionais e reunir os esforços da universidade no contexto da pandemia.

Considerando que as universidades públicas, assim como a UNILAB, exercem um papel ímpar na sociedade, entendemos que uma de suas missões em tempo de crise é manter mecanismos de comunicação e disseminação de informação junto à comunidade acadêmica. Portanto, consideramos válido o esforço da produção de uma comunicação investigativa, como essa que realizamos, bem como dar publicidade a elas.

O objetivo do presente estudo foi conhecer as percepções e expectativas dos universitários do curso de Administração Pública da UNILAB a respeito dos efeitos da pandemia da COVID-19 em suas rotinas sem aulas na universidade. Para tanto, realizamos a coletada de dados primários por meio de um questionário on line, detalhado a seguir no tópico Desenvolvimento "Os caminhos da pesquisa".

Justificamos o interesse pela realização de tal estudo por entendermos que seus resultados poderão contribuir para encaminhamentos futuros por parte da universidade, ou para subsidiar a própria coordenação do curso que compôs a amostra estudada.

Vale ainda pontuar, que o presente estudo gira em torno das percepções e expectativas dos universitários do curso de administração pública da UNILAB. Portanto, os resultados aqui apresentados dão voz a esses estudantes, uma vez que dá visibilidade as mudanças ocorridas em suas vidas escolares ocasionadas pela pandemia.

\section{Desenvolvimento}

I. Os caminhos da pesquisa

Esse estudo é de caráter exploratório e abordagem quantitativa (DIEHL, 2004), adotando como procedimento para levantamento dos dados uma Enquete com aplicação de questionário estruturado (SAMPIERI et al, 20I3). A Enquete versou a respeito de questões objetivas que permitiram responder as quatro perguntas norteadoras da pesquisa: I) Que mecanismos os universitários estão 
Percepção e expectativas universitárias em tempo da COVID-19: um estudo de caso

utilizando para se informar sobre a pandemia da COVID-I9?, 2) Quais atividades estão realizando nesse momento de suspensão das aulas?, 3) Como os universitários estão lidando emocionalmente com a atual dinâmica de sociabilidade em período de quarentena?, e 4) O que os universitários vislumbram como forma de continuidade da sua formação educacional superior?

O questionário da Enquete foi elaborado na plataforma virtual Google Forms e seu acesso se deu por meio de um link. Posteriormente tal link foi encaminhado para o coordenador do curso de Administração Pública da UNILAB solicitando que o repassasse aos universitários do curso. Vale pontuar, que a escolha do curso se deu pelo fato da autora ter parceria acadêmica com uma docente do mesmo.

Como forma de garantir o anonimato dos universitários participantes da pesquisa, optou-se no momento do envio do link pela não identificação do e-mail dos participantes no momento do preenchimento do questionário na plataforma on line. Vale ressaltar, que no corpo da apresentação da Enquete, a pesquisadora criou um campo no formulário para que o universitário que tivesse de acordo com o objetivo da Enquete declarasse ciência e concordância em participar da pesquisa. A amostra da pesquisa foi então totalizada com 57 universitários participantes.

O questionário ficou disponível para acesso através do link durante o período de 28 de maio a 06 de junho. Participaram da pesquisa 57 universitários.

Foi utilizado o programa Excel Office no Windows 10 para realização da estatística descritiva dos dados obtidos por meio do questionário.

\section{Resultados e Discussão}

A amostra da pesquisa abarcou $57(n=100 \%)$ universitários, o que corresponde a $15 \%$ do total dos matriculados no Curso de Administração Pública, portanto, 379 universitários (UNILAB, 2019).

Referente à Questão I, que solicitava ao universitário informar se conhecia alguém contagiado pelo coronavírus e o grau de familiaridade com essa pessoa, essa foi respondida por 49 universitários, os quais indicaram "conhecer alguém", e somente 8 universitários declararam "não conhecer”. O grau de familiaridade declarada pelos 49 universitários foi: 21 afirmaram que tinham pouco contato com a pessoa infectada, 21 universitários afirmaram que a pessoa se tratava de parente, e apenas 7 indicaram que se tratava de amigo próximo.

Para a Questão 2 "Além das notícias apresentadas pelas TVs, você buscou alguma fonte de informação sobre o COVID-19?", 7 universitários afirmaram não buscar outra fonte de informação. Já 50 universitários declararam buscarem outras fontes de informação. Desses 50, 31 universitários responderam a Questão 3: "Qual tipo de fonte(s) de Informação?”, sendo que 13 indicaram obter informação via "site da prefeitura da Cidade que reside e site do Ministério da Saúde - Governo 
Federal". Já 10 universitários afirmaram buscar informação no "site da prefeitura da Cidade que reside e via rádio da Cidade". E 8 universitários indicaram obterem informação pela via "site da prefeitura da Cidade que reside ".

$\mathrm{Na}$ Tabela I, se observa as demais tipologias de informação indicadas pelos universitários. Os dados apontam que a maioria dos universitários demostram não se contentar somente com o que é comunicado pela mídia televisionada, e buscam outras fontes, como as divulgadas pelo governo federal e prefeituras.

Tabela I. Sistematização dos Tipos de Informação (Questão 3)

\begin{tabular}{lr}
\hline Tipo de fonte(s) de Informação & n \\
\hline Informação via site da prefeitura da Cidade que resido e Informação via site & 13 \\
do Ministério da Saúde - Governo Federal & 10 \\
$\begin{array}{l}\text { Informação via site da prefeitura da Cidade que resido e Informação via rádio } \\
\text { da Cidade que resido }\end{array}$ & 9 \\
Informação via site da prefeitura da Cidade que resido & 7 \\
Informação via site do Ministério da Saúde - Governo Federal & 3 \\
Informação via site da UNILAB & 3 \\
$\begin{array}{l}\text { Informação via site do Ministério da Saúde - Governo Federal e Informação } \\
\text { via site da UNILAB }\end{array}$ & 2 \\
$\begin{array}{l}\text { Informação via site da prefeitura da Cidade que resido e Informação via site } \\
\text { da UNILAB }\end{array}$ & Informação via site do Ministério da Saúde - Governo Federal e Informação \\
via site de outras Universidades & I \\
Informação via jornal impresso da Cidade que resido & | \\
Informação via site de outras Universidades & I \\
Informação via rádio da Cidade que resido e Informação via site de outras \\
Universidades
\end{tabular}

Fonte: Elaborada pela autora, 2020.

No Gráfico I, listamos os dez "Tipos de Sentimentos" declarados pelo universitários ao responderem a Questão 4 do questionário, que foi: Durante esse período de quarentena qual o Sentimento mais presente em sua vida?. Na distribuição de frequência (n) constatamos que a maioria, 12 universitários declararam o sentimento de "Tristeza", "Ter Dinheiro" foi declarado por II participantes. Já o sentimento de "Solidariedade" foi afirmado por 8 universitários. Em quarto no 
ranque, o sentimento "Ser Produtivo" foi apontado por 6 participantes, e em quinto lugar o sentimento de "Prevenção" apontado por 5. Os demais sentimentos elencados aparecem citados no Gráfico I.

A Questão 5, solicitava que o universitário indicasse a principal atividade estivesse realizando durante o seu dia a dia nesse período de quarentena, ou seja, de suspensão das atividades presenciais na universidade. Para tanto, um rol de oito tipos de atividades foram apresentadas aos participantes, como consta no Gráfico 2. Observa-se que a atividade declarada por um maior número de universitários foi a de "Serviços Caseiros" declarada por 18 universitários. Já "Leituras" foi declarada por 16 participantes, e 7 apontaram "Lazer". Em quarto lugar no ranque aparece "Trabalhos Acadêmicos" indicado por 5 universitários. A opção "Outros: criação do próprio negócio; cuidados com o pai; estudando para concurso público" foi declarada por 3 universitários, e as três últimas atividades indicadas por apenas dois participantes estão também indicadas no Gráfico 2, a seguir.

Gráfico I.Sentimento mais presente na vida dos universitários

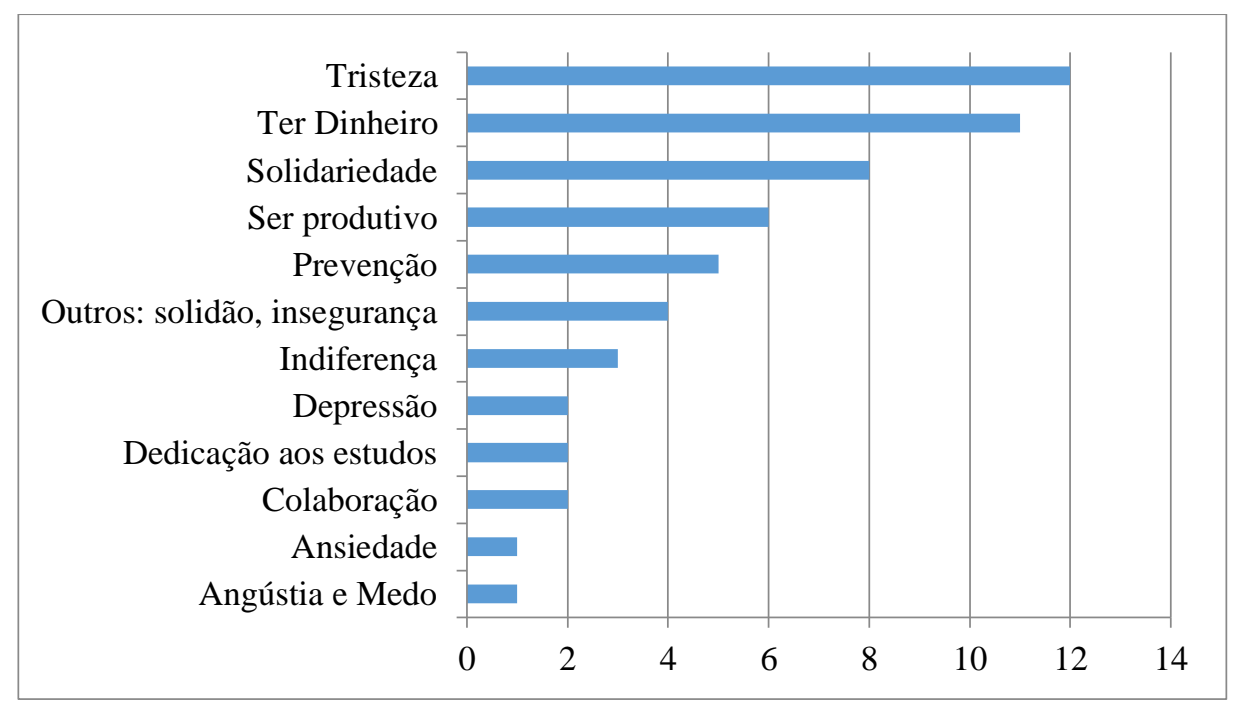

Fonte: Elaborado pela autora, 2020. 
Gráfico 2. Atividades desenvolvidas pelos universitários no dia a dia

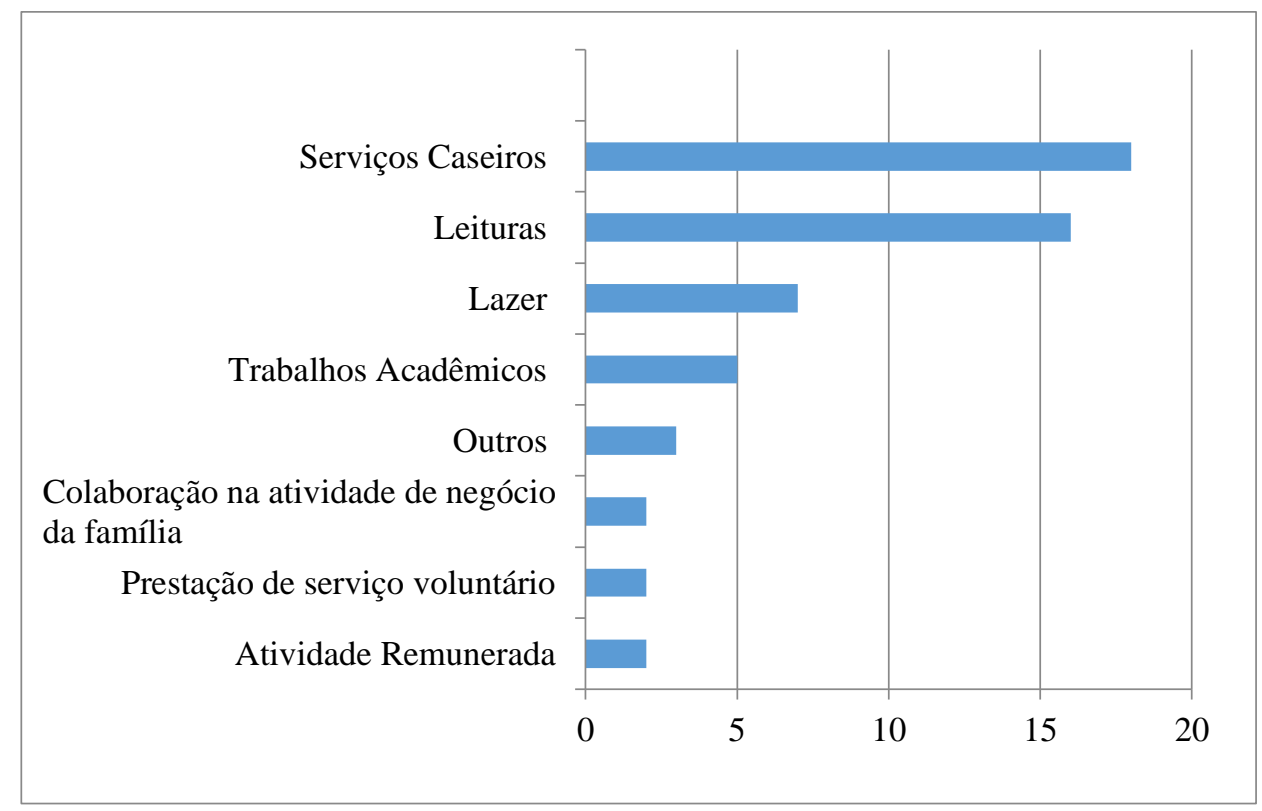

Fonte: Elaborado pela autora, 2020.

Os "Trabalhos Acadêmicos" foram apontados como a atividade principal por 5 universitários $(n=9 \%)$ do total de 57 universitários participantes da Enquete $(n=100 \%)$. Para esses 5 universitários Ihes foi solicitado que indicasse o "tempo" (quantidade de horas semanais) que dedicavam a atividade “Trabalhos Acadêmicos"(Questão 6), bem como indicar a "tipologia" desse "Trabalho Acadêmico" (Questão 7). Em respostas as duas questões apresentadas, respectivamente, os 5 universitários declararam "menos de I hora semanal a 9 horas semanais". Já referente a tipologia, 4 universitários declararam "Elaboração de Trabalho de Conclusão de Curso - TCC" e I "Artigo Científico".

No Gráfico 3, a seguir, o maior percentual de indicações em resposta à Questão 8 "Que aspecto da sua vida está mais afetado negativamente pela Pandemia do COVID-19?" foi referente a: "Esporte”, 23 universitários, "Financeiro" apontados por 15 universitários; e o terceiro aspecto mais indicado foi "Lazer" apontado por 10 universitários. Seguidos de: Relações familiares, Amorosa, Tempo dedicado ao estudo e Trabalho, cujos quantitativos de indicações por universitários foram respectivamente: 3,3,2 I . 
Percepção e expectativas universitárias em tempo da COVID-19: um estudo de caso

Gráfico 3. Os aspectos da sua vida mais afetados negativamente

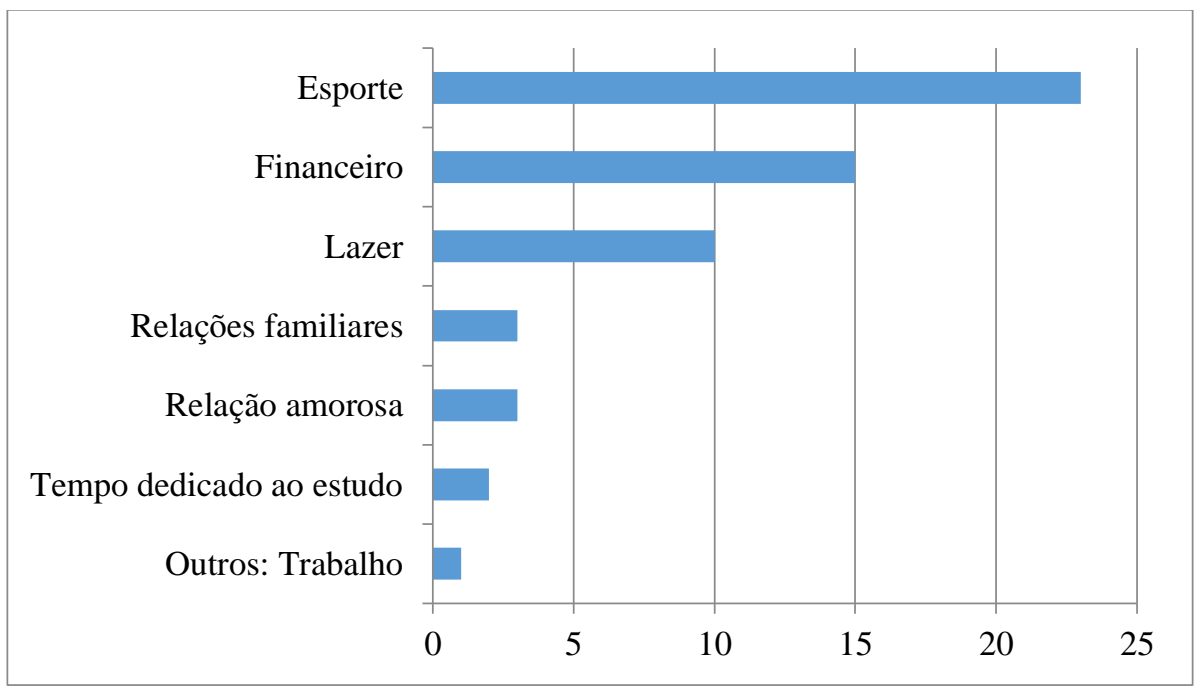

Fonte: Elaborado pela autora, 2020.

Na Tabela 2, abaixo, apresentamos as sugestões indicadas em resposta a Questão 9 "Quais suas sugestões para a continuidade das suas atividades acadêmicas do seu curso em período de COVID19?. Uma lista de sugestões foi apresentada aos universitários para que marcassem as que julgassem serem adequadas. Posteriormente as sugestões foram ranqueadas por número que vezes que foram indicadas pelos universitários em resposta a referida questão. Em primeiro lugar do ranque aparece como sugestão a "Oferta de disciplinas à distância", em segundo "Obrigatoriedade de uso de máscara durante a permanência na UNILAB". Como terceira sugestão do ranque consta: "Determinação de regras especificas de comportamento social da comunidade acadêmica dentro da UNILAB.

Tabela 2. Sistematização das sugestões (Questão 9)

\begin{tabular}{lc}
\multicolumn{1}{c}{$\begin{array}{c}\text { Sugestões para a continuidade das suas } \\
\text { atividades acadêmicas }\end{array}$} & $\begin{array}{c}\text { Número de } \\
\text { vezes indicadas }\end{array}$ \\
\hline $\begin{array}{l}\text { Oferta de disciplinas à distância (virtualmente). } \\
\text { Obrigatoriedade de uso de máscara durante a } \\
\text { permanência na UNILAB. }\end{array}$ & 29 \\
$\begin{array}{l}\text { Determinação de regras especificas de comportamento } \\
\text { social da comunidade acadêmica dentro da UNILAB } \\
\text { Higienização adequada nos ambientes físicos, sistema de } \\
\text { ar condicionados dos ambientes da UNILAB. }\end{array}$ & 16 \\
$\begin{array}{l}\text { Diminuir a quantidade de alunos por sala em todos os } \\
\text { cursos da UNILAB. }\end{array}$ & 15 \\
\hline
\end{tabular}

Fonte: Elaborada pela autora, 2020.

Olhar de professor, Ponta Grossa, v. 24, p. I-II, e-16144.052, 202 I.

Disponível em <https://revistas2.uepg.br/index.php/olhardeprofessor> 
Observam-se ainda na Tabela 2, duas outras sugestões também indicadas: "Higienização adequada nos ambientes físicos, sistema de ar condicionado e de refrigeração de água para beber"; e "Diminuir a quantidade de alunos por sala em todos os cursos da UNILAB".

\section{Considerações finais}

A Universidade Pública é sem sombra de dúvida o locus do diálogo para se pautar decisões de ordem política educacional que garantam a manutenção do ensino superior. Nesse momento que estamos impedidos de promover o diálogo presencial, fazer uso da tecnologia para viabilizar a produção e reprodução da comunicação se mostra bastante frutífera.

O presente ensaio é uma contribuição para a informação da comunidade acadêmica do curso de Administração Pública da UNILAB, bem como para os gestores da universidade. Informações essas que poderão vir a auxiliar no planejamento e na tomada de decisão sobre uma futura agenda de atividades acadêmicas.

De forma bastante resumida temos a pontuar a seguinte síntese às respostas das questões que nortearam nossa pesquisa: I) os universitários demonstraram ter espírito investigativo sobre a doença COVID-19, não se atendo apenas ao que é divulgado pelas mídias televisivas, 2) nesse momento de suspensão das atividades acadêmicas presenciais os universitários demonstram estarem mais voltados a realização de atividades laborais do que as de caráter intelectual, 3) com base nas narrativas dos universitários até o momento da realização da coletada das informações, ou seja, quase três meses após a paralização das atividades educacionais os mesmos demonstra que suas rotinas de sociabilidade estão alteradas, o que nos leva a crer que muito se deve ao fato de estarem cumprindo o distanciamento social, Outro aspectos é que a maioria os universitários declararam como sendo o sentimento que mais os influenciam no atual contexto é de Tristeza. Ao nosso ver está o relacionado diretamente a situação de calamidade que o Estado do Ceará vem sofrendo, que são mais de $4 \mathrm{mil}$ mortes causadas pelo coronavírus, e 4) a expectativa dos universitários relativa a continuidade da sua formação educacional superior que é a universidade oferte disciplinas on line até que voltem as aulas presenciais.

Por fim, ressaltamos que estudos de percepção devem ser considerados como instrumento que possibilitam uma melhor compreensão sobre processos sociais vividos e sentidos, como é este com nossos universitários, e que se bem utilizados contribuem para um planejamento mais humanizado por parte da UNILAB.

Olhar de professor, Ponta Grossa, v. 24, p. I-II, e-16|44.052, 202 I. 


\section{Referências}

BRASIL. Ministério da Saúde. Painel de casos de doença pelo coronavírus 2019 (COVID-19) no Brasil. Brasília. 2020. Disponível em: https://covid.saude.gov.br/. Acesso em I 3 de junho de 2020.

CEARÁ. Decreto $\mathbf{n}^{\circ} \mathbf{3 3 . 5} 10$ de 16 de março de 2020. Decreta situação de emergência em saúde e dispõe sobre medidas para enfrentamento e contenção da infecção humana pelo novo coronavírus. Fortaleza, 16 de março de 2020. Disponível em: https://coronavirus.ceara.gov.br/project/decreto-no33-5 I0-de-16-de-marco-de-2020/. Acesso em I3 de junho de 2020.

CEARÁ. Decreto $\mathbf{n}^{\circ} \mathbf{3 3 . 6 1 7}$ de 06 de junho de 2020. Prorroga o isolamento social no Estado do Ceará, renova a Política de Regionalização das medidas de isolamento social, e dá outras providências. Fortaleza, 06 de junho de 2020. Disponível em: https://www.seplag.ce.gov.br/wpcontent/uploads/sites//4/2020/06/DECRETO-N\%C2\%BA33.6I7-FASE-I.pdf. Acesso em I3 de junho de 2020.

DIEHL, Astor Antonio. Pesquisa em ciências sociais aplicadas: métodos e técnicas. São Paulo: Prentice Hall, 2004.

RODRIGUEZ-MORALES, Alfonso J.; GALLEGO, Viviana; ESCALERA-ANTEZANA, Juan Pablo; MÉNDEZ, Claudio A.; ZAMBRANO, Lysien I.; FRANCO-PAREDES, Carlos; SUÁREZ, Jose A.; RODRIGUEZ-ENCISO, Hernan D.; BALBIN-RAMON, Graciela Josefina; SAVIO-LARRIERA, Eduardo; RISQUEZ, Alejandro; CIMERMAN, Sergio. "COVID-19 in Latin America: The implications of the first confirmed case in Brazil". Travel Medicine and Infectious Disease, 101613, 2020. Disponível em: https://doi.org/10.1016/j.tmaid.2020.101613.

SÃO PAULO. Secretaria de Saúde do Estado de São Paulo. Boletim Epidemiológico COVID-I9. São Paulo. 2020. Disponível http://www.saude.sp.gov.br/resources/cve-centro-de-vigilanciaepidemiologica/areas-de-vigilancia/doencas-de-transmissaorespiratoria/boletim/boletim06_covid I9_070420.pdf. Acesso em 04 de junho de 2020.

SAMPIERI, Roberto Hernández; COLLADO, Carlos Fernández; LUCIO, María Del Pilar Baptista (orgs). Metodologia de pesquisa. Porto Alegre: Penso, 2013.

SES-SP. Boletim Epidemiológico Covid-1 9 - 07/04/2020. Governo do Estado de São Paulo, Secretaria de Estado da Saúde, Centro de Vigilância Epidemiológica "Prof. Alexandre Vranjac", 7 de abril de 2020. Disponível em: http://www.saude.sp.gov.br/resources/cve-centro-de-vigilanciaepidemiologica/areas-de-vigilancia/doencas-de-transmissaorespiratoria/boletim/boletim06_covid I9_070420.pdf. Acesso em 04 junho de 2020.

UNILAB. Portaria Reitoria $\mathbf{n}^{\circ} \mathrm{I} I 0$ de 17 de março de 2020. Dispõe sobre a instituição e designação dos membros responsáveis pelo Comitê de Enfrentamento ao COVIDI9(CIEC/UNILAB). Disponível em: http://www.unilab.edu.br/wpcontent/uploads/2020/03/SEI_23282.403880_2020_I9I.pdf. Acesso em I3 de junho de 2020.

UNILAB. Dados do Semestre 2019.2 Graduação. Disponível em: https://app.powerbi.com/view?r=eyjrljoiNTkzZjY2MWQtNjMzNSOOMjkzLWI4YTAtOGjjY2NmNjdm NzI I liwidCI6ljkwMjlkZGNILWFmMTItNDJiZS04MDM3LTU4MzEzZTRkYzVkMSJ9. Acesso em I4 de junho de 2020. 
WHO. Coronavirus desease (Covid- 19). Situation Report - 133. World Health Organization (WHO), $I^{\circ}$ de junho de 2020. Disponível em: https://www.who.int/docs/defaultsource/coronaviruse/situation-reports/2020060 I-covid-19-sitrep-133.pdf?sfvrsn=9a56f2ac_4. Acesso em 04 de junho de 2020.

\section{Agradecimentos}

A Professora Doutora Andrea Kanikadan, docente adjunta no curso de Administração Pública da UNILAB, pela sempre parceira em pesquisas, e por propiciar o contato com a coordenação do curso, viabilizando assim o envio do questionário on-line aos universitários.

Recebido em: 16 de junho de 2020.

Versão corrigida recebida em: 08 de dezembro de 2020.

Aceito em: 08 de dezembro de 2020.

Publicado online em: 04 de junho de 2021.

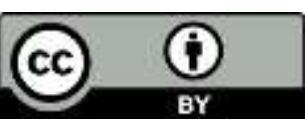

